Elsevier Editorial System(tm) for Nuclear Inst. and Methods in Physics Research, A

Manuscript Draft

Manuscript Number: NIMA-D-06-00361R2

Title: Radiation Tolerant Isolation Amplifiers for Temperature Measurement

Article Type: Review Article

Section/Category: Electronics

Keywords: COTS; Isolation amplifiers; Radiation damage.

Corresponding Author: Mrs. YI ZONG, PhD

Corresponding Author's Institution: University Complutense of Madrid

First Author: YI ZONG, PhD

Order of Authors: YI ZONG, PhD; FRANCISCO J. FRANCO, PhD; ANA C. FERNADEZ, PhD; JOSE G. MARQUES; JUAN A. de AGAPITO, PhD

Manuscript Region of Origin:

Abstract:

This paper concentrates on the selection of radiation tolerant isolation amplifiers, which are suitable for the signal conditioners for cryogenic system in the Large Hadron Collider (LHC). The evolution and the results of different commercial isolation amplifiers' parameters under neutron and gamma radiation are presented. In most cases, the tested isolation amplifiers' input offset voltage, bias currents and output offset voltage hardly changed during the radiation. The DC gain in input stage was only affected for some isolation amplifiers with a small open loop gain. Transmission coefficient showed decrease for all the tested isolation amplifiers. Also, the $\mathrm{DC}$ output voltage increased and the ripple voltage decreased for all the build in isolated regulators. In addition, results on 1B41 signal conditioner showed that it was tolerant to $7-8 \cdot 10^{12} \mathrm{n} / \mathrm{cm}^{2}$, which was $50 \%$ higher than the expected dose in the LHC. 


\title{
Radiation Tolerant Isolation Amplifiers for Temperature Measurement
}

\author{
Yi Zong ${ }^{\mathrm{a}^{*}}$, Francisco J. Franco ${ }^{\mathrm{a}}$, Juan A. de Agapito ${ }^{\mathrm{a}}$, Ana C. Fernández \\ José G. Marques ${ }^{\mathrm{b}}$ \\ ${ }^{a}$ Departamento de Física Aplicada III,Facultad de Ciencias Físicas, Universidad Complutense \\ de Madrid,Ciudad Universitaria,28040 MADRID (SPAIN) \\ ${ }^{b}$ Portuguese Research Reactor,Instituto Tecnológico e Nuclear, Estrada Nacional, 10 \\ 2686-953 SACAVEM (PORTUGAL)
}

\section{ABSTRACT}

This paper concentrates on the selection of radiation tolerant isolation amplifiers, which are suitable for the signal conditioners for cryogenic system in the Large Hadron Collider (LHC). The evolution and the results of different commercial isolation amplifiers' parameters under neutron and gamma radiation are presented. In most cases, the tested isolation amplifiers' input offset voltage, bias currents and output offset voltage hardly changed during the radiation. The DC gain in input stage was only affected for some isolation amplifiers with a small open loop gain. Transmission coefficient showed decrease for all the tested isolation amplifiers. Also, the DC output voltage increased and the ripple voltage decreased for all the build in isolated regulators. In addition, results on 1B41 signal conditioner showed that it was tolerant to $7-8 \cdot 10^{12} \mathrm{n} / \mathrm{cm}^{2}$, which was $50 \%$ higher than the expected dose in the LHC.

PACs: $29.90 .+\mathrm{r} \quad$ 28.52.Lf $28.41 . \mathrm{Rc}$

Keywords: COTS; Isolation amplifiers; Radiation damage.

\footnotetext{
* Correspond ing author, Yi Zong is with the Departamento de Fisica Aplicada III, Facultad de Ciencias F is ic as, Universidad Complutense de Madrid, 28040 Madrid (SPAIN), on leave from the Wuhan Institute of Techno logy, Wuhan (China). Tel.: 34-913944441, fax: 34-913945196, E-mail address: yzong@fis.ucm.es
} 


\section{INTRODUCTION}

Isolation amplifiers are used as analog interfaces between systems with separated grounds. They prevent most high voltages from reaching an instrument's low-voltage inputs, protecting the equipment. Isolation amplifiers also break ground loops removing commonmode voltages from a measurement circuit. They can amplify or attenuate input signals of many ranges to create signals with a common voltage range, and they also can provide isolated DC power for active sensors [1].

The temperature of the High Temperature Sensors (HTS) leads in the cryogenic system of the Large Hadron Collider (LHC) will be measured by means of large numbers of PT-100 RTDs. The working temperature of the HTS leads is supposed to be between $40 \mathrm{~K}$ and $300 \mathrm{~K}$. Therefore, the value of PT-100 resistance will vary approximately between $10 \Omega$ and $100 \Omega$. The allowed uncertainty margin is up to $1.5 \Omega$. Moreover, the DC voltage between HTS leads and ground might reach $1900 \mathrm{~V}$. It is impractical to run a separate ground wire for each sensor in this case. Furthermore, safety regulations often require local ground connections. Electronic isolation amplifiers are clearly suitable for solving the measurement problem, but the selection of isolation amplifiers must be carefully done since a neutron fluence of $5 \cdot 10^{12} \mathrm{n} / \mathrm{cm}^{2}$ and a total gamma dose of $100 \mathrm{~Gy}(\mathrm{Si})$ are expected for a 10-year activity period in the LHC cryogenic system.

The isolation between the input and output stages is usually done by means of three kinds of devices: Op tocouplers, coupling transformers or capacitors [2]. Nevertheless, optical coupling isolators were immediately discarded because of the well-known sensitivity of these devices to displacement damage [3]. Besides, the use of the capacitive coupling amplifiers should be avoided due to the fact that they usually need the external regulators, this option being not recommendable. Considering the above reasons, in this paper, we will focus on the description of the isolation amplifiers using transformers coupling. 


\section{DESCRIPTION OF TESTED ISOLATION DEVICES}

All the tested devices were manufactured by Analog Devices (AD), transformers coupling being the isolation system preferred by AD. The set of tested candidates is shown in Table I.

Although the complete datasheets of the tested supervisory circuits can be obtained from the web site of the corresponding companies, a brief description will be given here. The tested $\mathrm{AD}$ devices are included in the category of isolation amplifiers. This means that the input stage is an isolated operational amplifier with external passive component, such as resistors and capacitors, connected to a modulator that transmits the op-amp output value to the other stage through a coupling transformer, as shown in Fig. 1. The AD202 and AD204 are gen eral purpose, two-port, transformer-coupled isolation amplifiers that may be used in a broad range of applications, where input signals must be measured, processed, and/or transmitted without a galvanic connection. The main difference between the AD202 and the AD204 is that the AD202 is powered directly from a $15 \mathrm{~V} \mathrm{DC}$ supply while the AD204 is powered by an externally supplied clock, such as the recommended AD246 clock driver.

The $\mathrm{AD} 210$ is the latest member of a new generation of low cost, high performance isolation amplifiers. The AD210 is powered by a single $+15 \mathrm{~V}$ supply. The three-port design structure permits the AD210 to be applied as an input or output isolator, in single or multichannel applications with high accuracy and complete galvanic isolation. The AD210 interrupts ground loops and leakage paths, and rejects common-mode voltage and noise that may degrade measurement accuracy. In addition, its topology provides protection from fault conditions that may cause damage to other sections of a measurement system.

The AD215 is a high speed input isolation amplifier designed to isolate and amplify wide bandwid th analog signals. It provides complete galvanic isolation between the input and 
output of the device including user-available front-end isolated power supplies.

All of the above Analog Devices' isolation amplifiers AD2XY have one same advantage. That is they eliminate the need for an external, user-supplied dc-to-dc converter. This permits the designer to minimize the necessary circuit overhead and consequently reduce the overall design and component costs.

The 1B41 is a precision, isolated, RTD signal conditioner that incorporates a circuit design utilizing transformer based isolation. It is especially suitable for harsh environments with extremely high common mode interference. 1B41 generates its own floating current excitation, providing true low cost channel-to-channel isolation. Based on this kind of devices, 1B41 signal conditioner can measure the value of a PT-100 resistor in the interval of temperatures $\left[\mathrm{T}_{\mathrm{L}}, \mathrm{T}_{\mathrm{H}}\right]$ (Fig. 2). This device provides an output voltage with a value proportional to the difference between $\mathrm{R}_{\mathrm{TD}}$ (PT-100 sensor) and $\mathrm{R}_{\mathrm{Z}}$, reference resistor with a value equal to $\mathrm{R}_{T D}$ at $\mathrm{T}=\mathrm{T}_{\mathrm{L}}$.

\section{IRRADIATION FACILITY}

All the tested isolation amplifiers were irradiated in the neutron facility of the Portuguese Research Reactor (Lisbon, Portugal). The Reactor Portuguese of Investigation (RPI) is an open-pool, light-water moderated and cooled reactor, with $1 \mathrm{MW}$ thermal power. A dedicated irradiation facility was built around beam tube E4, consisting of a dry irradiation chamber with dimensions of $100 \mathrm{~cm} \times 60 \mathrm{~cm} \times 60 \mathrm{~cm}(1 \times \mathrm{w} \times \mathrm{h})$ at the end of the tube, as well as a cylindrical prolongation inside the beam tube, $100 \mathrm{~cm}$ long and with $150 \mathrm{~mm}$ inner diameter [4]. The neutron beam has a diameter of $150 \mathrm{~mm}$ in both the chamber and its prolongation. For biological shielding purposes, layers of $\mathrm{Cd}$ lined polyethylene and high density concrete are used. The arrangement of the layers allows the use of relatively short connecting cables (ca. $4 \mathrm{~m}$ ) to the measuring instruments. 
The neutron spectrum in the irradiation facility is essentially a leakage spectrum in a light-water moderated ${ }^{235} \mathrm{U}$ fission reactor. While most fission neutrons are in the $1-2 \mathrm{MeV}$ energy range, there are still neutrons with energy in excess of $10 \mathrm{MeV}$, although these contribute with only about $0.1 \%$ of the total neutron flux above $1 \mathrm{MeV}$. The gamma field in the irradiation cavity was reduced through a $40 \mathrm{~mm}$ thick $\mathrm{Pb}$ filter, in order to obtain a neutron/photon ratio close to the one expected for the LHC. An additional $7 \mathrm{~mm}$ thick boral $\left(\mathrm{B}_{4} \mathrm{C}-\mathrm{Al}\right)$ filter was inserted next to the $\mathrm{Pb}$, in order to reduce the thermal neutron component and thu s the activation of circuits and cables.

The spectrum in the irradiation cavity was simulated using the Monte-Carlo code MCNP-4C, using a detailed three-dimensional model of the core validated with extensive measurements in the core region [5]. The calculated spectrum was adjusted to measurements using sets of activated foils with different energy-dependent cross sections, following a procedure previously described [6]. Fig. 3 shows the adjusted spectrum per lethargy unit, inside the irradiation chamber, at the point closest to the core. For comparison, a spectrum corresponding to a core position close to the entrance of the beam tube is also shown. A strong decrease in the low energy component is evident. Table II shows neutron dosimetry values at the point closest to the core.

The fast neutron fluence during the irradiations was measured with $\mathrm{Ni}$ foils activated through the ${ }^{58} \mathrm{Ni}(\mathrm{n}, \mathrm{p}){ }^{58} \mathrm{Co}$ nuclear reaction. The ${ }^{58} \mathrm{Co}$ isotope has a half-life of $70.78 \mathrm{~d}$, which is convenient for an activation lasting several days. The fluences obtained in this way are $40 \%$ higher than the total fluence above $1 \mathrm{MeV}$ for the neutron spectrum in the irradiation cavity, as determined in ref.[6].

The fluences in the irradiation of electron ic devices are normally expressed in terms of a $1 \mathrm{MeV}$ neutron equivalent neutron fluence for Si. The hardness parameter in the irradiation 
cavity was determined using the Si damage data of ref. [7] and the adjusted spectrum. Fig. 4 shows the differential hardness parameter as function of the neutron energy. The energy intervals are the same as used for the adjusted spectrum. Through the combination of the low neutron flux intensities for low energies in the cavity and the low cross section for damage in that region, the main contribution for the hardness parameter comes from the $0.2-8 \mathrm{MeV}$ energy interval. Following the procedure of ref. [7], the hardness parameter in the 0.01-12 $\mathrm{MeV}$ energy interval was determined to be $0.81(5)$.

All the fluences in the results below are expressed in terms of the activated Ni foils. The equivalent $1 \mathrm{MeV}$ fluence is obtained multiplying these values by 1.27 , taking in to account that it is $78 \%$ higher than the total flux above $1 \mathrm{MeV}$ and thus $27 \%$ higher than the fluence determined with the Ni foils.

Since the vestigial gamma doses are several times higher than the expected value of gamma radiation, the tests are valid as NIEL tests and as accelerated TID tests, as CERN irradiation protocol suggests [8].

\section{TEST SET-UP}

All the isolation amplifiers from Analog Devices (Fig. 1) can be divided into several sections: Isolated operational amplifier, isolated power supplies and output stage. Therefore, the characterization of the whole isolation amplifier is carried out in the following three parts:

Parameters of the op amp: Like any other operational amplifiers, those present in the isolated stage have an input offset voltage, $\mathrm{V}_{\mathrm{IOS}}$, and input bias currents, $\mathrm{I}_{\mathrm{B}^{+}} \& \mathrm{I}_{\mathrm{B}-}$. Their values can be calculated from the voltages at the output FB and the inverting \& non-inverting inputs at several specific feedback networks. Also, it is very interesting to describe the transfer function between $\mathrm{V}_{\mathrm{IN}}$ and $\mathrm{V}_{\mathrm{FB}}$ by means of the DC gain, which depends on the 
external resistors, the open loop gain, and the typ ical output error.

Isolated internal regulators: Mean and ripple voltages are the main parameters of this section as well as the output current.

Transmission efficiency: The voltages of FB and OUT in an ideal isolation amplifier are equal. However, in a real one, the relationship is more accurately described with the expression $\mathrm{V}_{\mathrm{OUT}}=\mathrm{TC} \cdot \mathrm{V}_{\mathrm{FB}}+\mathrm{V}_{\mathrm{OUT}}$, OS , where $\mathrm{TC}$ is the transmission coefficient (ideally 1 ) and $\mathrm{V}_{\text {OUT, os }}$ the offset voltage related to the output stage.

Finally, the special characteristics of 1B41 make its radiation tests a little bit different from other amplifiers. In this case, we preferred to test the device while it was measuring a known resistance and register its output during the irradiation. So, several devices were irradiated in order to know the output value connected to their input with different resistors between $10 \Omega$ and $100 \Omega$, playing the role of $\mathrm{R}_{\mathrm{TD}}$ sensors.

In any case, the measure of each parameter requires different resistor networks. This problem was solved by using several mechanical relays that, after switching, could change the feedback network of the device. Tested printed circuit boards (PCBs) were connected to the measuring system, which consisted of a PC, an I-V source measure unit, an $81 / 2$ digits multimeter and a switch system with 40 -channel multiplexer cards and $4 \times 10$ matrix cards. The control program is performed by the object-oriented language Testpoint ${ }^{\circledR}[9]$. During the irradiation, the PC system checked the main parameters of the devices every ten minutes and their values were stored in several files with date and time for a later study. Unfortunately, some parameters could not be measured during the irradiation (e.g., the parameters of frequency response, etc.) so they were eventually measured one month after the irradiation when the devices could be safely handled. Meanwhile, devices were unbiased and stored at 
room temperature.

\section{EXPERIMENTAL RESULTS AND ANALYSIS}

\subsection{RESULTS ON AD2XY ISOLATION AMPLIFIERS}

During the irradiation all the devices were biased with $\pm 15 \mathrm{~V}$ power supplies. In order to select the best rad-tolerant device, two samples of each model were tested to determine the most tolerant one in the first campaign and, afterwards, the second campaign was centered on the study of the selected rad-tolerant device.

Besides, it is important to point out that all the values of the neutron fluences in this paper are measured by the Ni foils. In order to express it in the equivalent $1-\mathrm{MeV} \mathrm{n} \cdot \mathrm{cm}^{-2}$, these values should be multiplied by 1.27 , as explained in the Section III. In addition, the relationship between TID value and neutron fluence is that $16.47 \mathrm{~Gy}(\mathrm{Si})$ is equivalent to $10^{12}$ $\mathrm{n} \cdot \mathrm{cm}^{-2}$

\subsubsection{DC input parameters}

Input offset voltage and bias currents of the isolated operational amplifier are included in this category. Input offset voltage of all the devices hardly changes during the irradiation (Fig. 5), except AD215. As illustrated in Fig. 6, AD215 amplifier shows a great increase in this parameter.

Input bias currents of these amplifiers, except AD215, were not significantly affected by this radiation. AD215 suffered a large increase of these parameters, as Fig. 6 shows. It is interesting to point out the fact that the evolution of $\mathrm{I}_{\mathrm{B}}$ was similar for both samples as a function of the neutron fluence. On the contrary, there is no dependence on the TID value, so we conclude that the main damage cause is the displacement damage rather than the ionization. 


\subsubsection{DC gain in input stage}

This parameter was measured in two situations: First of all, the amplifier was configured as a buffer with unity gain and, finally, a pair of resistors with values of $22 \& 220$ $\mathrm{k} \Omega$ set the gain into a value about 11 . The edge values of the input data sweep were selected in order to obtain output data between $\pm 1 \mathrm{~V}$.

For an ideal operational amplifier, this parameter is only related to the value of feedback resistors, but for the real ones, there is a small dependence on the open loop gain. This parameter decreases as neutron fluence increases [10], but this is not a serious handicap for amplifiers with a high open loop gain (AD210, AD215). However, other amplifiers such as AD202 and AD204 have a quite low initial value so it was observed a decrease in the value of the DC gain. Indeed, the fact leads to the measured value of DC gain below the theoretical one. For example, an AD202 sample showed an input DC gain of 10.92, instead of the theoretical value, 10.97, after receiving the radiation value of $1.2 \cdot 10^{13} \mathrm{n} / \mathrm{cm}^{2} \& 198(\mathrm{~Gy})$.

\subsubsection{Transmission coefficient}

This parameter is the ratio between the output and input gain and it is related to the gains of the modulator/demodulator and output buffer. Ideally, its value is 1 although it is usually lower. All the amplifiers showed a decrease in this parameter, which is very significant in AD202 \& AD204 (Fig. 7).

\subsubsection{Output offset voltage}

Usually, this parameter hardly shifts during the irradiation. The only exception is $\mathrm{AD} 215$, where there is an increase about $5 \mathrm{mV} / 10^{12} \mathrm{n} / \mathrm{cm}^{2}$ (Fig. 8). As consequence of the failure of AD246, AD204 shows transitory discontinuities in Fig. 8.

\subsubsection{Typical output error}


It has been assumed that the isolation amplifier is completely linear when we dealt with the evolution of the gain value in the former sections. Nevertheless, this is not true. There is always a difference between the theoretical and the actual value.

Typical output error is defined as the highest difference between the actual value of $\mathrm{V}_{\text {OUT }}$ and the ideal one calculated from $\mathrm{V}_{\mathrm{IN}}$, DC gain, offset voltages, etc. which are used to estimate the non-linearity of the function $\mathrm{V}_{\text {OUT }}=f\left(\mathrm{~V}_{\mathrm{IN}}\right)$. It hardly changes during the irradiation so we deduce that there is no relationship between this parameter and the neutron fluence. The exact value depends on the DC gain, usually, the higher the gain, the higher the error value. Table III shows the average values measured during the irradiation.

\subsubsection{Isolated regulators}

During the irradiation, the mean \& r.m.s ripple voltages of each internal isolated power supply were measured. None was loaded with external resistors except the input operational amplifier. The initial output values were about $\pm 7-8 \mathrm{~V}$ for $\mathrm{AD} 20 \mathrm{X}$ and $\pm 15-16 \mathrm{~V}$ for $\mathrm{AD} 21 \mathrm{X}$.

There is a growth of the DC output value. For instance, an AD210 showed an increase

of $+\mathrm{V}_{\text {ISO }}$ from $16.2 \mathrm{~V}$ to $16.8 \mathrm{~V}$ when the neutron fluence reached the value of $10^{13} \mathrm{n} / \mathrm{cm}^{2} \&$ 166 (Gy). Similar shifts were found for the rest of the isolation amplifiers. The authors believe that this growth is associated with the decrease of the quiescent current of the operational amplifiers loaded with the internal regulator. The phenomenon was observed by different authors in other irradiated devices [11][12].

On the contrary, the ripple voltage decreases in all the devices. For example, the previous device changed from $325 \mathrm{mV}$ r.m.s to $250 \mathrm{mV}$ r.m.s after the irradiation.

\subsubsection{Evolution of $\mathrm{AD} 246$}


This device is an external clock driver whose purpose is to provide a $0-15 \mathrm{~V}$ square signal for AD204 supplying. During the irradiation, average and r.m.s values were measured to find out the tolerance of the device.

Due to the duty cycle is $50 \%$, both values must be ideally $7.5 \mathrm{~V}$. Fig. 9 shows the evolution of these parameters during the irradiation. We observed that the device suffers transitory disruptions above $7-8 \cdot 10^{12} \mathrm{n} / \mathrm{cm}^{2}(116-133(\mathrm{~Gy}))$. The consequences of these strange disruptions were also found in the output of AD204. We really do not know the exact reason why the failure appeared.

In addition, after the off-line measurement, we found that the parameters of frequence response did not change for the all above tested isolation devices under the radiation environment.

\subsection{RESULTS ON 1B41 SIGNAL CONDITIONER}

Six samples of this device were irradiated while they were measured with reference resistors of 10,47 and $100 \Omega$. During the irradiation, they were also biased with $\pm 15 \mathrm{~V}$ power supplies.

We found that these devices are hardly affected by the radiation below $7-8 \cdot 10^{12} \mathrm{n} / \mathrm{cm}^{2}$ (113-133(Gy)) (Fig. 10). If it exceeds this level, the output voltage value begins to increase very quickly and, finally, the device stops working when the neutron fluence reaches a different value for every sample. For example, a device could not tolerate a neutron fluence of $9 \cdot 10^{12} \mathrm{n} / \mathrm{cm}^{2}(147(\mathrm{~Gy}))$ although another one could survive till $1.8 \cdot 10^{13} \mathrm{n} / \mathrm{cm}^{2}(298(\mathrm{~Gy}))$. 
The most tolerant parts of the device are the internal current sources $\mathrm{I}_{\mathrm{RTD}} \& \mathrm{I}_{\mathrm{Z}}$, which can tolerate a neutron fluence above $1.5 \cdot 10^{13} \mathrm{n} / \mathrm{cm}^{2}(247(\mathrm{~Gy}))$ and, eventually, these parts fail at $2.5 \cdot 10^{13} \mathrm{n} / \mathrm{cm}^{2}(413(\mathrm{~Gy}))$.

According to our tests, the lower the values of the resistors, the more accurate the measurements are. This fact might be related to a shift of the DC gain value of the internal stages, whose effect would be negligible with very low input voltages, but more and more important as the input voltage increases.

\section{AD210 AS A RTD SIGNAL CONDITIONER}

From the experimental data, we may conclude that the most tolerant devices are $\mathrm{AD} 210$ and 1B41. However, the former device was characterised by measuring the typical electrical parameters $\left(\mathrm{V}_{\mathrm{OS}}, \mathrm{I}_{\mathrm{B}}, \mathrm{G}_{\mathrm{OL}}\right.$, etc. $)$, and the latter was tested while measuring a resistor with a known value. Therefore, it will be interesting to compare both devices supposing they were tested in the same conditions. To do that, we imagined that a PT-100 RTD was biased with a $250-\mu \mathrm{A}$ current source, as it actually happens for the 1B41, and that the voltage drop along the temperature sensor was amplified by an AD210 so that the output levels should be similar to those of the $1 \mathrm{~B} 41$.

We can calculate the theoretical resistance of a PT-100 sensor as a function of the temperature, according to the following quadratic equation (1):

$$
\mathrm{R}=\mathrm{R}_{0} \times\left(1+\alpha \mathrm{T}+\beta \mathrm{T}^{2}\right)
$$

Where $\alpha=3.9 \cdot 10^{-3}, \beta=-5.85 \cdot 10^{-7}$. Supposing that the value of $\mathrm{R}_{0}$ is $100 \Omega, 47 \Omega$ and $10 \Omega$, respectively, we can estimate the error observed in the output stage of the AD2 10 while measuring the temperature. Accepting that the offset voltage and the gain error have been previously removed (for instance, with potentiometers), the mathematical calculations show 
that the error is within the uncertainty margin up to a radiation value of $1.79 \cdot 10^{13} \mathrm{n} / \mathrm{cm}^{2}$ (295(Gy)). In other words, this kind of device would work with accuracy even if it is exposed to a total radiation dose three times more than that expected during 10 years of LHC activity. In fact, another paper accounts for the results shown in this paper [13].

\section{CONCLUSION}

The results obtained in the first campaign show that some isolation devices are not suitable for the specific use in the LHC cryogenic system. These devices and reasons to refuse them are the following:

AD202 \& AD204: Decrease in open loop gain and degradation in the modulator/demodulator buffer.

AD215: Large increase in input bias currents, input and output offset voltage.

AD246: External clock driver necessary for AD204 fails at $7 \cdot 10^{12} \mathrm{n} / \mathrm{cm}^{2}$.

According to our tests, $1 \mathrm{~B} 41$ can tolerate a total radiation dose of $7-8 \cdot 10^{12} \mathrm{n} / \mathrm{cm}^{2} \&$ 116-133(Gy), which is $50 \%$ higher than the expected dose. Also, the AD210 isolation amplifier could be an adequate candidate to be used in the LHC cryogenic system because of two reasons: An acceptable tolerance to radiation and a set of very good characteristics. For both devices, a later radiation test on six samples belonging to different batches confirmed the results shown in previous sections.

\section{ACKNOWLEDGEMENTS}

This work was supported by the cooperative agreement K746/LHC between CERN \& UCM and by the Spanish Research Agency CICYT (FPA2002-00912). We would like to express out the appreciation to the operators at the Portuguese Research Reactor for their assistance during the irradiation campaigns. 


\section{REFERENCES}

[1] M. Rowe, Isolation boosts safety and integrity, Test \& Measurement World, August, 2002, http:/www.reed-electronics.com/tmworld/article/CA235286

[2] A. J Peyton, V. Walsh, Analog Electronic with Op Amps: a source book of practical circuits, Cambridge Univ ersity Press, Cambridge, UK, 1993

[3] G. Messenger, M. Ash, The effects of radiation on electronic systems, New York, Van Nostrand Reinhold, Second Edition, 1992

[4] J. G. Marques, A. C. Fernandes, I. C. Gonçalves and A. J. G. Ramalho, Proceedings of the 5th Workshop on Radiation Effects on Components and Systems, Madrid, 2004, Eds. Elisa Rangel Prudencio, Remo Tamayo González and Carmen Sánchez de la Fuente (Instituto Nacional de Técnica Aeroespacial, Madrid, 2004, ISBN 84-930056-14) pp. 335-338.

[5] A.C. Fernandes, I.C. Gonçalves, N.P. Barradas and A.J. Ramalho, Nucl. Tech. 143 (2003) 358.

[6] A.C. Fernandes, I.C. Gonçalves, J.G. Marques, J. Santos, A.J.G. Ramalho and M. Osvay, Mixed-field dosimetry of a fast neutron beam at the portuguese research reactor for the irradiation of electronic circuits - measurements and calculations, Proceedings of the 11 th International Symposium on Reactor Dosimetry, Brussels, 2002, Eds. J. Wagemans, H. A. Abdherrahim, P. D'hondt, C. De Raedt, World Scientific (Singapore, 2003) pp. 143-149.

[7] Standard practice for characterizing neutron energy fluence spectra in terms of an equivalent monoenergetic neutron fluence for radiation hardness testing of electronics, ASTM Stand ard E722-04, 2004.

[8] ATLAS standard radiation test methods http:/atlas.web.cern.ch/Atlas/GROUPS/FRONTEND/radhard.htm.

[9] http://www.keithley.com/products/software/?mn=TESTPOINT

[10] G C. Messenger and M. S. Ash, The effects of radiation on electronic systems, $2^{\text {nd }}$ Edition, New York, Van Nostrand Reinhold, 1992.

[11] A. H. Johnston, IEEE Transactions on Nuclear Science, 23(1976), 1709.

[12] M. Menichelli et al., IEEE Tran sactions on Nuclear Science, 47(2000), 1879.

[13] V. Remondino, Radiation damage in amplifiers used for quench detection in a superconducting accelerator, $3^{\text {rd }}$ European Conference on Radiation and its Effects on Components and Systems, 18-22 September. 1995, pp. 89-93. 


\section{FIGURE CAPTIONS}

Fig. 1: Typical structure of Analog Devices' isolation amplifiers. Coupling transformers allow the communication between isolated stages. Additional resistors determine the DC gain of the system.

Fig. 2: Simplified internal topo logy of 1B41 signal conditioner. The gain of the differential amplifier is determined by external resistors. Isolated voltage sources, present in the actual device, are not included in this schema.

Fig. 3: Neutron spectrum per lethargy unit, inside the irradiation chamber, at the point closest to the core. The spectrum corresponding to the core is also shown for comparison.

Fig. 4: Differential hardness parameter as function of neutron energy

Fig. 5: Evolution of $\mathrm{V}_{\mathrm{IOS}}$ in several $\mathrm{AD}$ isolation amplifiers. Peaks appeared in the dashed line for AD204 are related to transitory failures of the external clock driver, AD246.

Fig. 6: Evolution of DC input parameters of AD215 as a function of neutron fluence. The peaks correspond to the reactor annealing period.

Fig. 7: Transmission coefficient in AD's isolation amplifiers. Discontinuities in AD204 plot are related to AD246 failures.

Fig. 8: Output off set voltage, $\mathrm{V}_{\mathrm{OUT}}$, os, in AD's amplifiers.

Fig. 9: Evolution of AD246 output during the irradiation. For unknown reason AD246 undergoes transitory failure when the radiation dose is greater than $7-8 \cdot 10^{12} \mathrm{n} / \mathrm{cm}^{2} \& 116$ 133(Gy).

Fig. 10: Temperature measured by $1 \mathrm{~B} 41(\mathrm{RTD}=100 \Omega)$. Dashed lines are the exact temperature that had to be determined. 


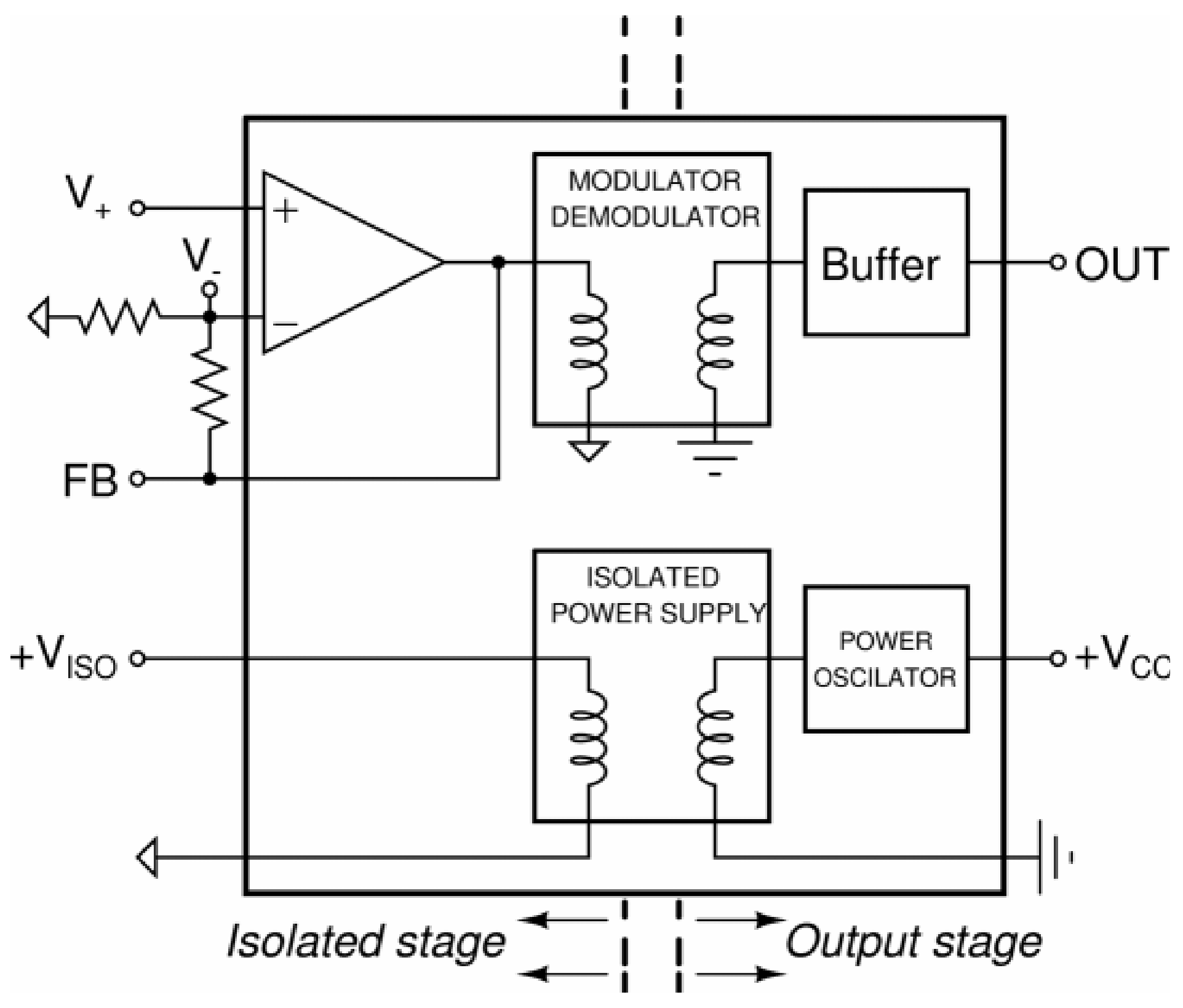

FIG. 1 


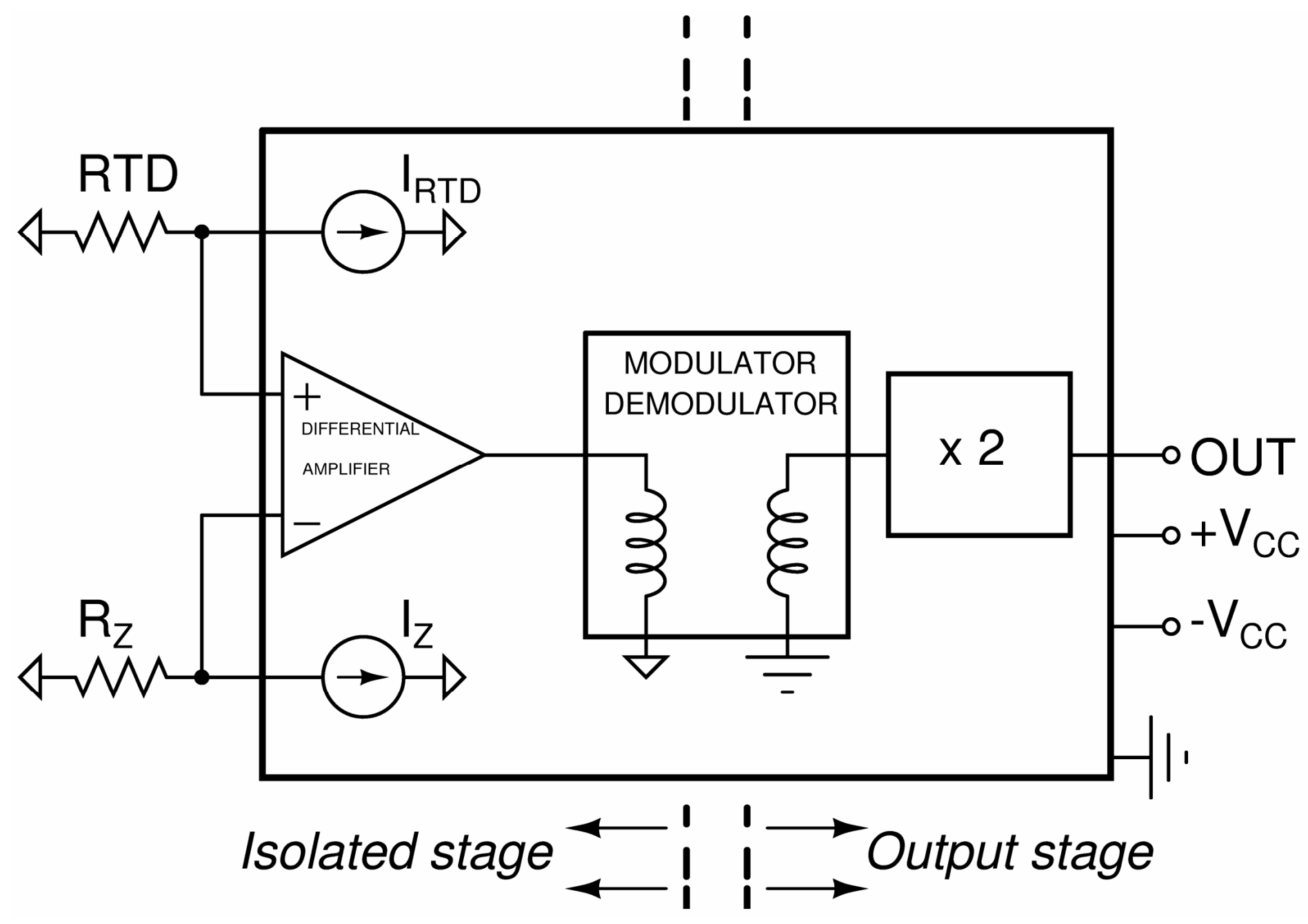

FIG. 2 


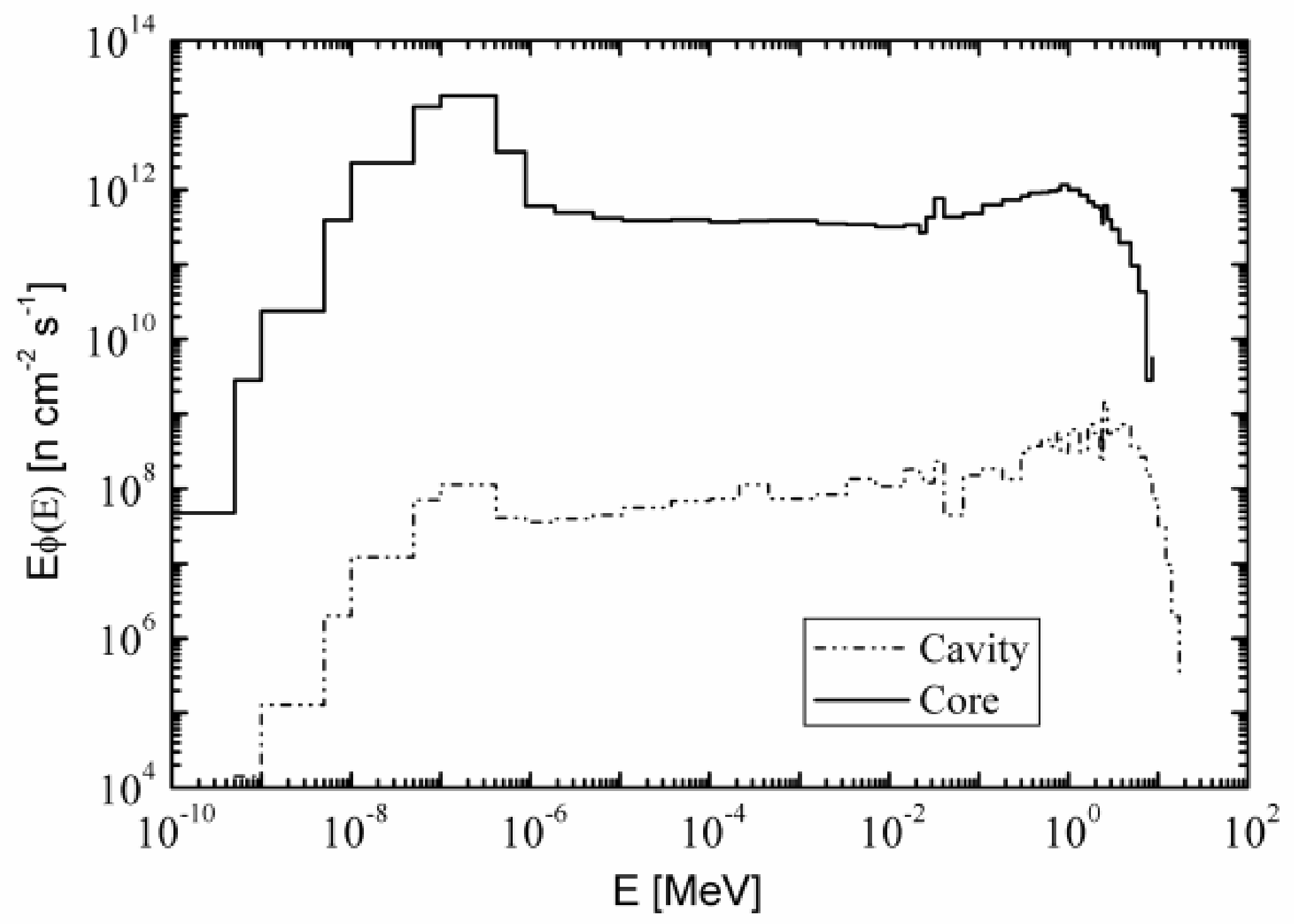

FIG. 3 


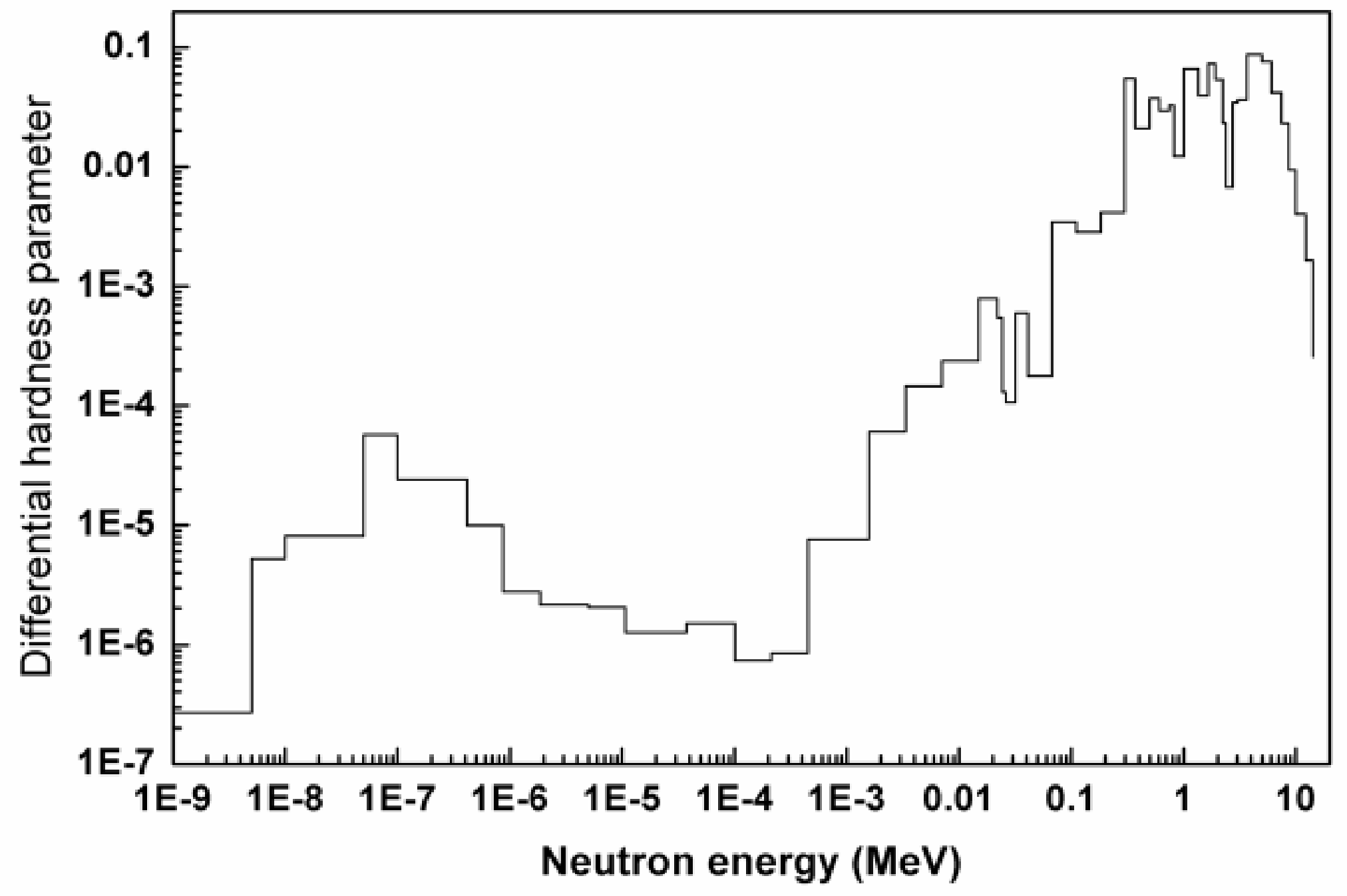

FIG. 4 


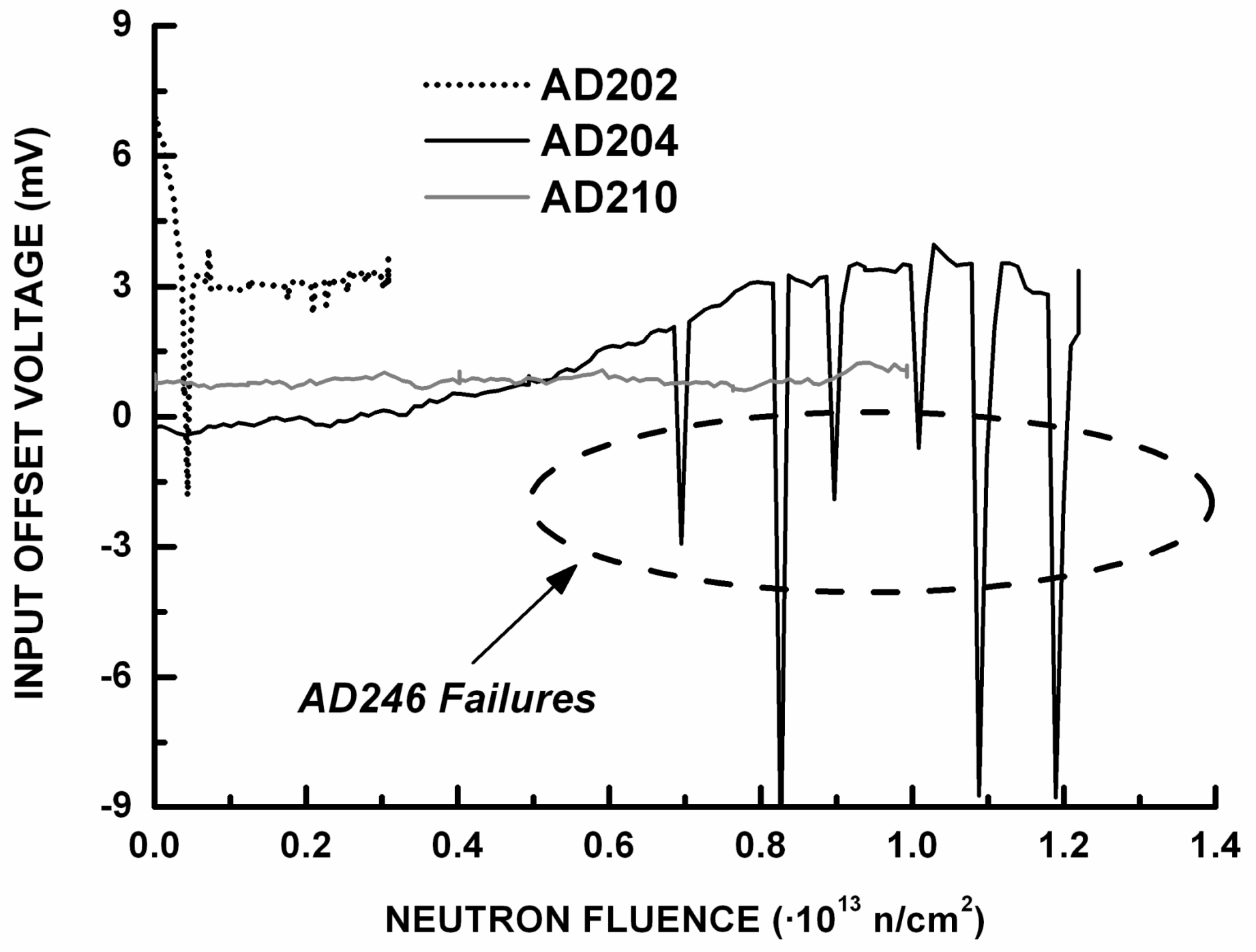

FIG. 5 


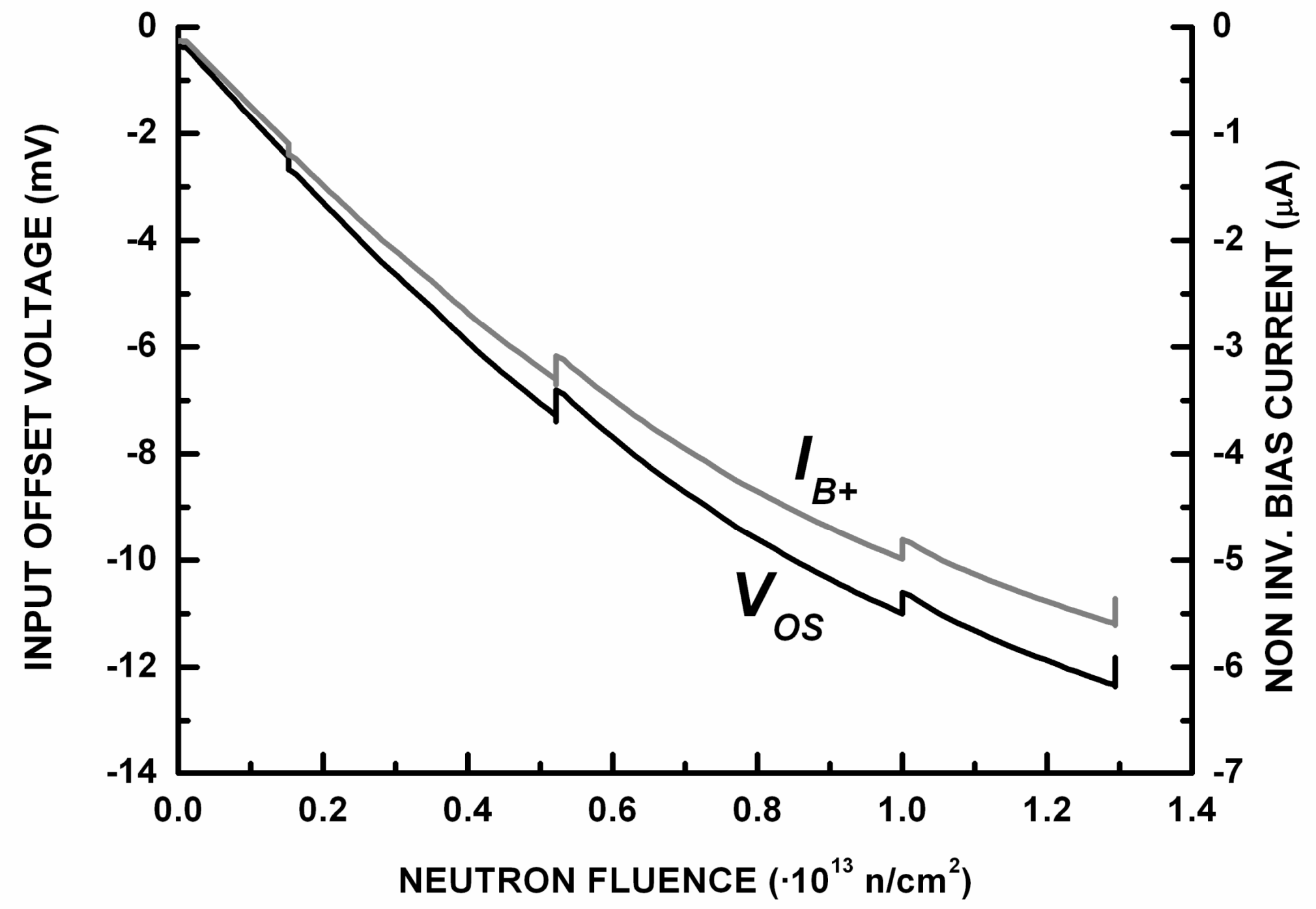

FIG. 6 


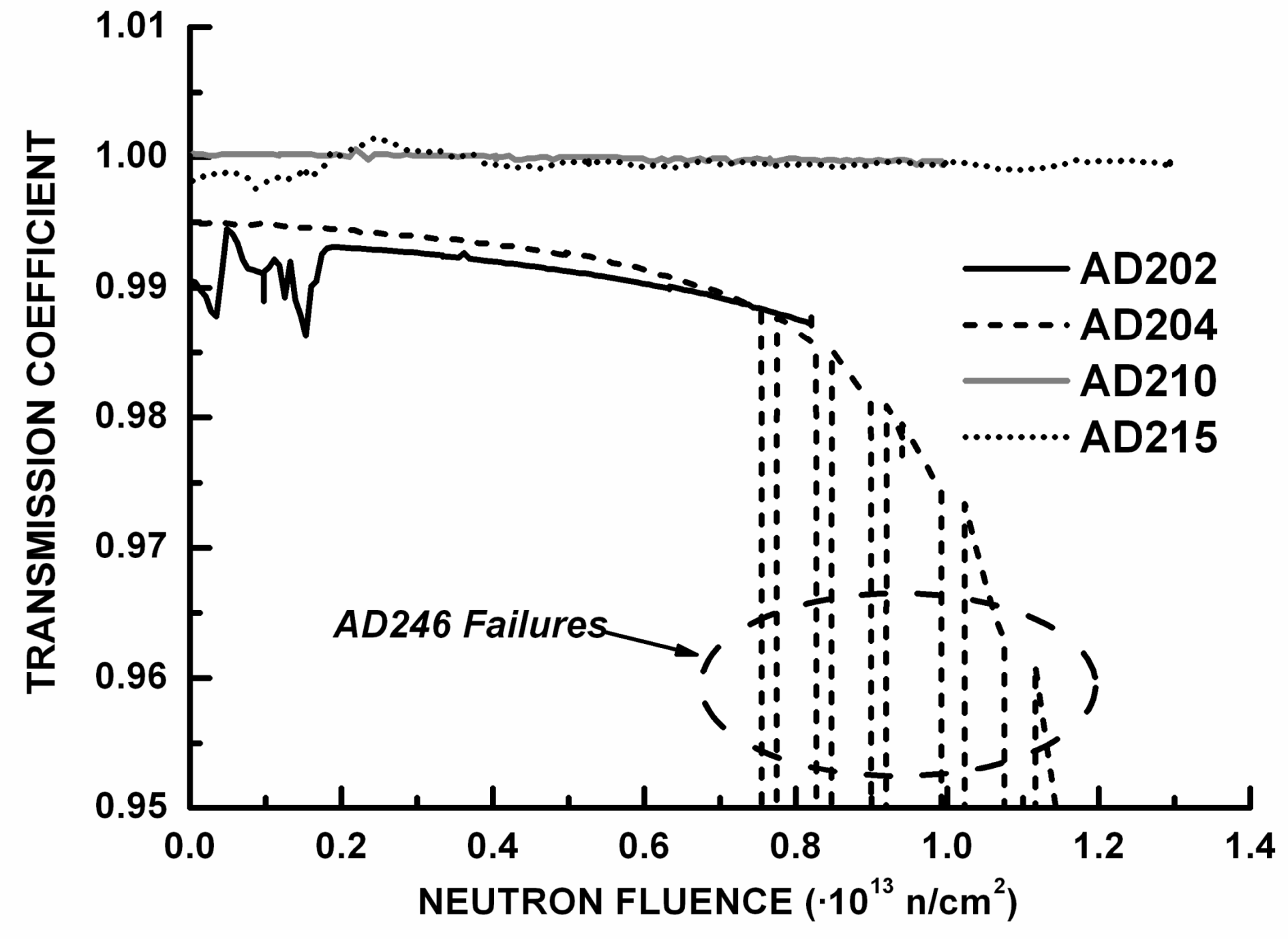

FIG. 7 


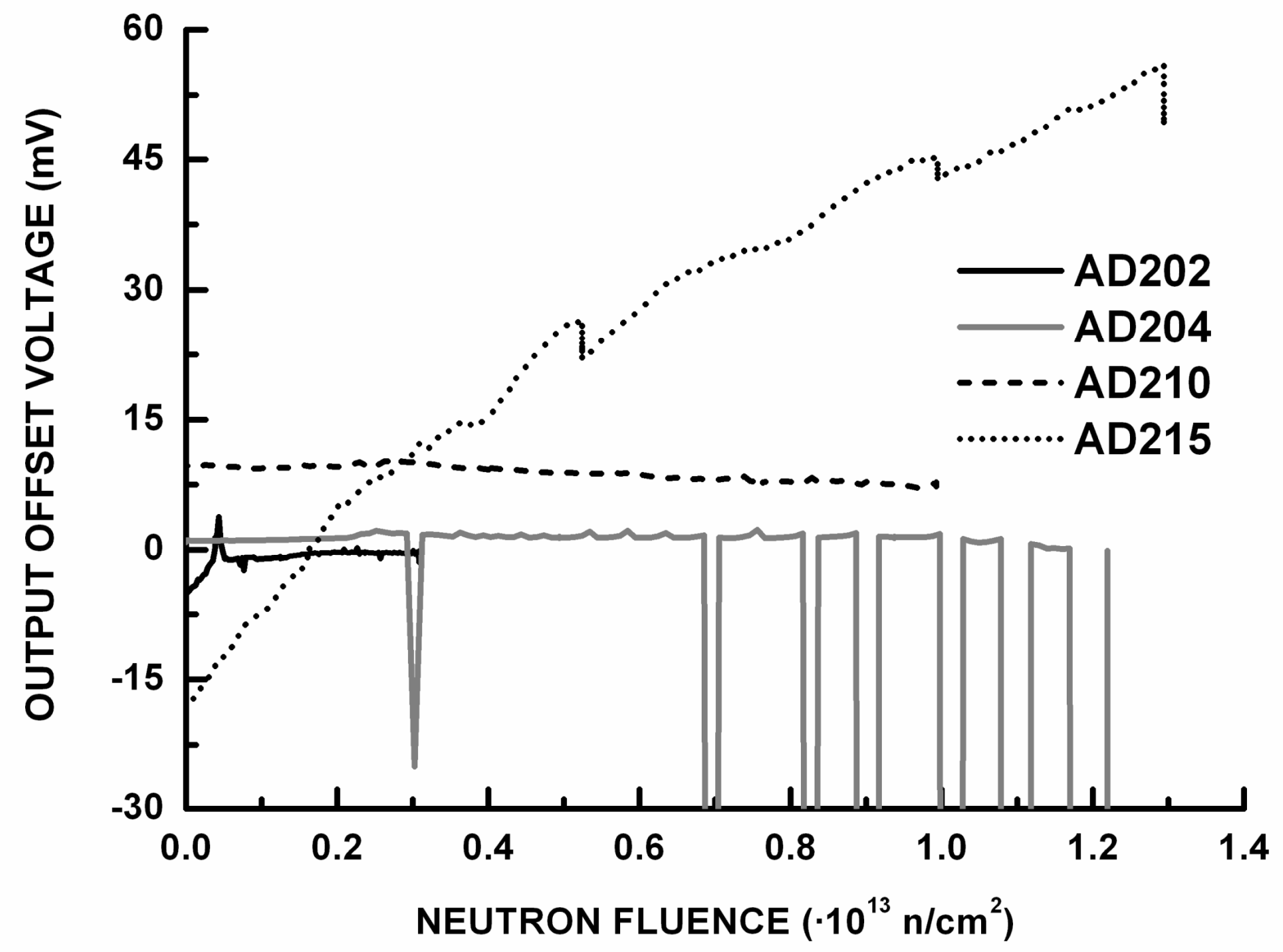

FIG. 8 


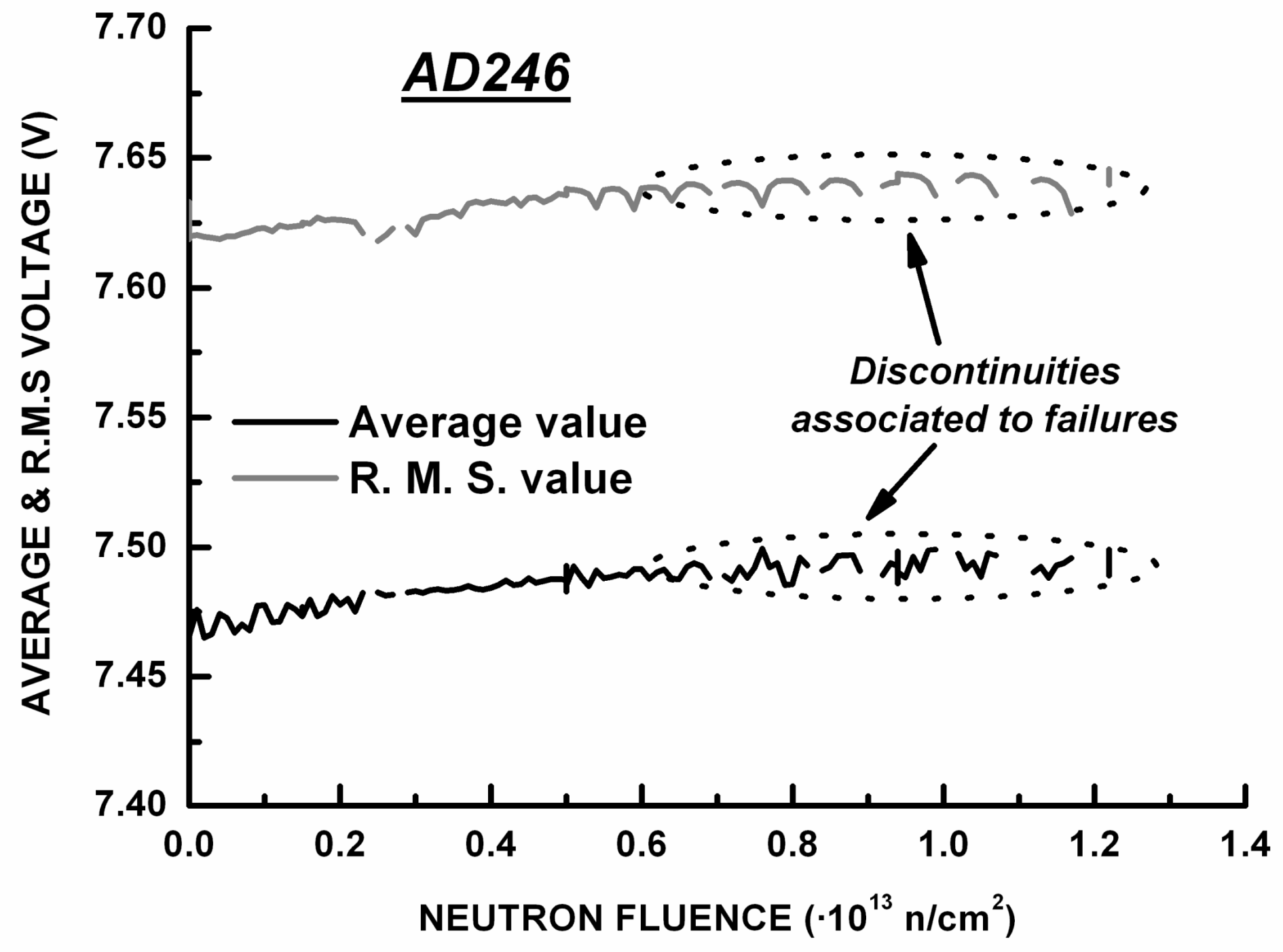

FIG. 9 


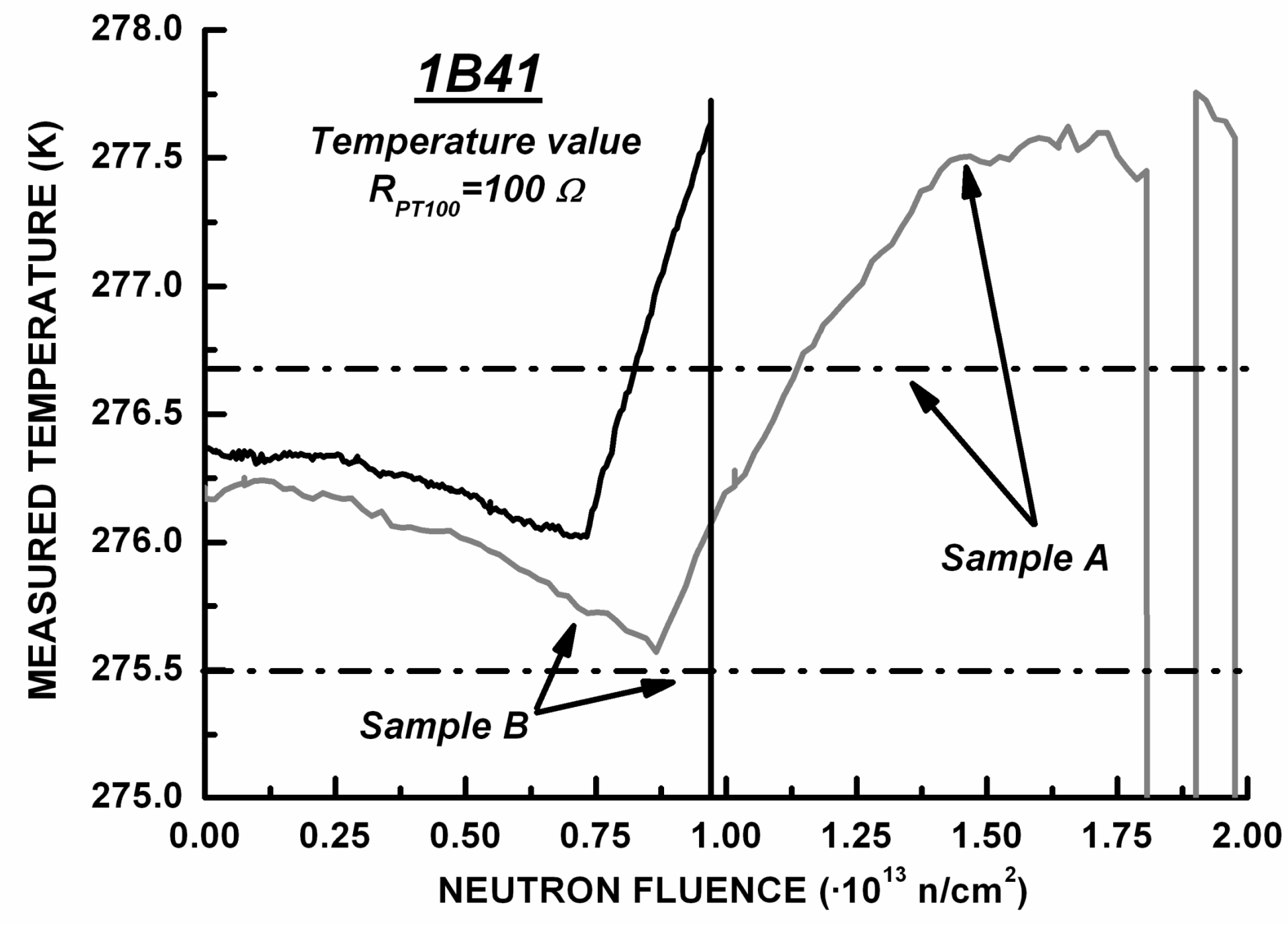

FIG. 10 
Table I: Tested isolation devices

\begin{tabular}{cccccc}
\hline \hline Model & Manufacturer & Kind & $\begin{array}{c}\text { Internal Isolated } \\
\text { Regulators? }\end{array}$ & $\begin{array}{c}\text { DC } \\
\text { Isolation }\end{array}$ & Main Characteristics \\
\hline AD202 & $\mathrm{AD}$ & $\begin{array}{c}\text { Isolation } \\
\text { amplifier }\end{array}$ & Yes, $\pm \mathrm{V}_{\mathrm{ISO}}$ & $\pm 2000 \mathrm{~V}$ & Low cost, miniature, unipolar supply \\
AD204 & $\mathrm{AD}$ & $\begin{array}{c}\text { Isolation } \\
\text { amplifier }\end{array}$ & Yes, $\pm \mathrm{V}_{\mathrm{ISO}}$ & $\pm 2000 \mathrm{~V}$ & $\begin{array}{c}\text { Similar to AD202 but with external } \\
\text { clock signal, pro vided by AD246(also } \\
\text { te sted) }\end{array}$ \\
$\mathrm{AD} 210$ & $\mathrm{AD}$ & $\begin{array}{c}\text { Isolation } \\
\text { amplifier }\end{array}$ & $\begin{array}{c}\text { Yes, } \pm \mathrm{V}_{\mathrm{ISO}} \\
\& \pm \mathrm{V}_{\mathrm{OSS}}\end{array}$ & $\pm 3500 \mathrm{~V}$ & Precision, wide band width, 3 -port \\
$\mathrm{AD} 215$ & $\mathrm{AD}$ & $\begin{array}{c}\text { Isolation } \\
\text { amplifier } \\
\text { Signal }\end{array}$ & $\mathrm{Yes}$ & $\pm 1500 \mathrm{~V}$ & $120 \mathrm{kHz}$ band width, low distortion \\
1B41 & $\mathrm{AD}$ & Yes & $\pm 1500 \mathrm{~V}$ & Very few additional external devices \\
\hline \hline
\end{tabular}

Table II. Neutron dosimetry parameters

\begin{tabular}{cc}
\hline \hline Parameter & Value \\
\hline Thermal neutron flux, $\mathrm{E}<0.5 \mathrm{eV}\left(\mathrm{n} \cdot \mathrm{cm}^{-2} \cdot \mathrm{s}^{-1}\right)$ & $2.7 \times 10^{8}$ \\
Epithermal neutron flux, $\mathrm{E}=1 \mathrm{eV}\left(\mathrm{n} \cdot \mathrm{cm}^{-2} \cdot \mathrm{s}^{-1}\right)$ & $4.0 \times 10^{7}$ \\
Fast neutron flux, $\mathrm{E}>1 \mathrm{MeV}\left(\mathrm{n} \cdot \mathrm{cm}^{-2} \cdot \mathrm{s}^{-1}\right)$ & $1.0 \times 10^{9}$ \\
Fast neutron flux, Ni foils $\left(\mathrm{n} \bullet \mathrm{cm}^{-2} \cdot \mathrm{s}^{-1}\right)$ & $1.4 \times 10^{9}$ \\
Equivalent 1 MeV flux $\left(\mathrm{n} \cdot \mathrm{cm}^{-2} \cdot \mathrm{s}^{-1}\right)$ & $1.78 \times 10^{9}$ \\
Multiplication factor from Ni foils to $1 \mathrm{MeV}$ equivalent & 1.27 \\
Hardness parameter & 0.81 \\
\hline \hline
\end{tabular}

TABLE III: Typical error value of AD's iso lation amplifiers

\begin{tabular}{ccccc}
\hline \hline Model & $\begin{array}{c}\text { DC } \\
\text { Gain=1 }\end{array}$ & Gain drift & $\begin{array}{c}\text { DC } \\
\text { Gain=11 }\end{array}$ & Gain drift \\
AD202 & 0.3 & 30 & 1.3 & 130 \\
AD204 & 0.15 & 15 & 0.4 & 40 \\
AD210 & 0.03 & 1.5 & 0.1 & 5 \\
AD215 & 0.1 & 5 & 0.2 & 10 \\
& $\mathrm{mV}$ & ppm & $\mathrm{mV}$ & $\mathrm{ppm}$ \\
\hline \hline
\end{tabular}


All editorial requests are made.

* Response to Reviewers \&/or Editor

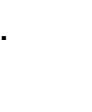

All editorial requests are made.

(1) 